\title{
Allergic rhinitis common in otitis media patients
}

Published online: 02 November 2012

(C) Springer Healthcare 2012

medwireNews: Allergic rhinitis is a common comorbidity in children with otitis media with effusion, suggest study findings.

Notably, there was no link between otitis media and mechanical nasal mucosal swelling, suggesting that allergy is the connecting factor, say the researchers.

"A likely mechanism is that an allergic inflammation in the respiratory epithelium at the entrance and inside the Eustachian tube can result in tube dysfunction due to swelling in this region and possibly cause a secondary inflammation in the middle ear," suggest study author Hans Bisgaard (University of Copenhagen, Denmark) and colleagues.

Writing in Clinical and Experimental Allergy, Bisgaard and team assessed 291 children, aged 6 years on average, who were participating in the Copenhagen Prospective Studies on Asthma in Childhood 2000 birth cohort study for the presence of otitis media with effusion and allergic rhinitis and related conditions.

Overall, $39 \%$ of the children in the cohort had otitis media with effusion and $10 \%$ had allergic rhinitis.

Children with otitis media were a significant 3.36-fold more likely to also have allergic rhinitis than children without the ear inflammation. Nasal mucosal swelling, nasal eosinophilia, nonallergic rhinitis, asthma, and eczema were not associated with the presence of otitis media, however.

There has been some controversy as to whether otitis media is linked with allergies or not, as only some studies have suggested a link, explain the researchers.

The results of this study are in agreement with those of previous research showing that the middle ear mucosa can produce an allergic response, they add.

"We therefore propose a link between otitis media with effusion and allergic inflammation of the middle ear," say Bisgaard and co-workers, although they acknowledge that "new studies are needed to assess and replicate these findings and confirm this proposed link."

By Helen Albert, Senior medwireNews Reporter

\section{Reference}

Clin Exp Allergy 2012; 42: 1615-1620 